\title{
Exploding dissipative solitons in reaction-diffusion systems
}

\author{
Orazio Descalzi, ${ }^{1,2, *}$ Nail Akhmediev, ${ }^{2,3}$ and Helmut R. Brand ${ }^{2}$ \\ ${ }^{1}$ Complex Systems Group, Facultad de Ingeniería y Ciencias Aplicadas, Universidad de los Andes, Av. Mons. Álvaro del Portillo 12.455, \\ Las Condes, Santiago, Chile \\ ${ }^{2}$ Department of Physics, University of Bayreuth, 95440 Bayreuth, Germany \\ ${ }^{3}$ Optical Sciences Group, Research School of Physics and Engineering, Institute of Advanced Studies, The Australian National University, \\ Canberra, ACT 0200, Australia
}

(Received 9 July 2013; revised manuscript received 1 October 2013; published 17 October 2013)

\begin{abstract}
We show that exploding dissipative solitons can arise in a reaction-diffusion system for a range of parameters. As a function of a vorticity parameter, we observe a sequence of transitions from oscillatory localized states via meandering dissipative solitons to exploding dissipative solitons propagating in one direction for long times followed by the reverse cascade back to oscillatory localized states. While exploding dissipative solitons are known from the cubic-quintic complex Ginzburg-Landau (CGL) equation, propagating exploding dissipative solitons appear to require for their existence a system of lower symmetry such as the reaction-diffusion model studied here.
\end{abstract}

DOI: 10.1103/PhysRevE.88.042911

PACS number(s): 82.40.Ck, 05.70.Ln, 47.20.Ky, 82.40.Bj

\section{INTRODUCTION}

Dynamics of reaction-diffusion (RD) systems is a well-established and growing area of modern research [1]. Complex dynamics in RD systems leads to a wide range of motions in the form of traveling waves, pattern formation, and emergence of more general types of self-organized structures. More recently, much attention has been paid to formation and dynamics of dissipative solitons (DS). The latter are localized structures appearing not only in reaction diffusion systems but in a much wider range of dynamical systems in nature [2] and in the laboratory [3]. This powerful concept led to significant progress in laser optics [4], in understanding biological systems [5], and even in nanotechnology [6].

In systems far from equilibrium, a continuous driving force and the resulting dynamics are the reasons for the existence of stationary structures. Dissipative solitons are an excellent example of this notion. DSs exist due to a complex balance between various physical effects [5]. In optics, this is the balance between energy pump and dissipation as well as between nonlinearity and dispersion [4]. In reaction-diffusion systems, the role of the balance between energy pump and dissipation is played by the balance between matter input and output. More intricate are systems that include both energy and matter flows within the same dissipative soliton [7].

DSs are found to have a number of complex dynamics. The simplest one is the structure with an envelope that is stationary despite the continuous flows of energy and/or matter occurring within the soliton. Another example is a pulsating dissipative soliton that periodically changes shape with the balances kept on average. Interaction of DSs [8,9] and their self-replication [10-12] may have far reaching consequences in explaining complex biological functions. Moreover, continuous matter and energy flows within the soliton can be

*odescalzi@miuandes.cl interpreted as the simplest form of metabolism, while their eventual disappearance can be considered as "death." Thus, dissipative solitons can be viewed as prototypes of the simplest organisms at the beginning of life evolution on Earth. In other words, they can explain the origin of life from the mix of chemicals in the presence of a continuous supply of energy from the sun.

Mathematically, DSs can be modeled by the complex cubicquintic complex Ginzburg-Landau (CGL) equation [13] and its generalizations. The CGL equation arises in a variety of situations in nonlinear optics as well as near the onset of a weakly inverted bifurcation to traveling waves in the case of pattern-forming instabilities. Among its stable solutions, there are localized pulses and holes of various types. Their properties and interactions have been extensively studied for one and two spatial dimensions [5,14-24].

There is a significant number of experimental observations of DS. These include the cases of binary fluid convection $[25,26]$, surface reactions [27], the Faraday instability in granular media [28] and colloidal suspensions [29], sheared electroconvection [30], and nonlinear optics [31-33]. For a review of transverse patterns in nonlinear optics we refer to Ref. [34]. Modeling and the experimental results on DS are usually well matched. A good example of such correspondence is the explanation of the partial annihilation of pulses observed in Refs. [25-27] as being due to small noise [24].

The most intriguing behavior of DSs are their explosions. They have been predicted theoretically $[35,36]$ and detected experimentally in nonlinear optics [37]. Recent studies of exploding dissipative solitons have focused on the bifurcation scenarios leading to exploding dissipative solitons [38-41], on the influence of noise on these rather unique objects [42] as well as on their generalization to two spatial dimensions [43-45]. It has been shown, in particular, that explosions can be azimuthally symmetric [45], as well as asymmetric. Asymmetric explosions may lead to their diffusive chaotic motion in a two-dimensional extended system.

We note that meandering is a signature of chaos for exploding dissipative solitons. After explosions, explosive 
DSs never recover their previous position [40,42]. As for the changes of directions of exploding DSs, the explosions are a big perturbation for the DS and because of parity symmetry of the underlying equations both directions are possible. The influence of additive noise on prototype equations for pattern formation, such as the Swift-Hohenberg equation or the cubic-quintic CGl equation, has been studied from various perspectives. In Ref. [46] the influence of thermal noise on the Swift-Hohenberg equation for various boundary conditions has been quantitatively analyzed. Spatial and temporal noise power spectra of stripe patterns for a stochastic Swift-Hohenberg equation have been studied in Ref. [47]. For the cubic-quintic Swift-Hohenberg equation, which allows for stable localized solutions of arbitrary lengths due to a trapping mechanism [48], it has been shown that noise leads to a finite lifetime since it induces a release of the localized state from the trap [48]. Noise effects can also determine the outcome of collisions between stable localized states as it has been demonstrated of collisions of counter-propagating pulses for coupled cubic-quintic CGL equations [24]. We finally mention that a small noise amplitude has a drastic influence on the transition to exploding dissipative solitons in the cubic-quintic CGL equation as already mentioned briefly above [42].

Explosions happen when the flow of energy and/or matter from internal regions of a soliton to its outskirts is too high to keep the flow being laminar [39]. Excessive amount of the energy (matter) flow may distort the soliton and may lead to a behavior that looks like real explosions. Exploding dissipative solitons are as generic as stationary or pulsating ones in the sense that they also occupy large areas in the multidimensional space of system parameters [36]. If we take this fact into account, the variety of exploding behavior of DSs is even more impressive than one would imagine initially.

Here we demonstrate that exploding dissipative solitons can also arise for simple reaction-diffusion equations for two variables and we show that in this type of equation, exploding dissipative solitons can propagate for long times, a behavior unknown from the cubic-quintic CGL equation because of its higher symmetry.

\section{THE MODEL}

For the two-component RD system, we use the following general model:

$$
\begin{gathered}
\partial_{t} u=\mu_{1} u-\mu_{2} v+\beta_{1} u^{3}+\gamma_{1} u^{5}-\beta_{2} v^{3}-\gamma_{2} v^{5} \\
+D_{r} u_{x x}-D_{i} v_{x x}, \\
\partial_{t} v=\mu_{3} v+\mu_{2} u+\beta_{1} v^{3}+\gamma_{1} v^{5}+\gamma_{2} u^{5} \\
\quad+D_{r} v_{x x}+D_{i} u_{x x},
\end{gathered}
$$

where $u(x, t)$ and $v(x, t)$ are chemical concentrations, $\beta_{1}$ is positive in order to guarantee that the bifurcation is subcritical, and $\gamma_{1}$ is negative in order to ensure saturation. The parameters $\mu_{1}$ and $\mu_{3}$ are negative since we will focus on subcritical behavior. The coefficient $\mu_{2}$ corresponds to the detuning in optics or to a vorticity parameter in fluids.

We will call it vorticity in the following. The diffusion coefficient $D_{r}$ and the linear dispersion $D_{i}$ are both positive. The parameters $\beta_{2}$ and $\gamma_{2}$ are nonvariational parameters associated with nonlinearities.

For the simulation of Eqs. (1) and (2) we use as numerical method explicit fourth-order Runge-Kutta finite differencing with a grid of 250 points in $x$ along a grid spacing of $d x=0.2$ (corresponding to a box size 50) and a time step $d t=0.01$. The stability of the solutions has been tested introducing a small amount of noise and waiting long enough to avoid transients (in some cases $T \sim 10^{5}$ ).

In our numerical simulations we keep all parameters fixed except for $\mu_{2}$, the vorticity. The parameter values that we used are $\mu_{1}=\mu_{3}=-0.2, \beta_{1}=1.0, \beta_{2}=1.2, \gamma_{1}=-0.1$, $\gamma_{2}=-0.9, D_{r}=0.125$, and $D_{i}=0.5$. A variety of stable solutions arises as a function of $\mu_{2}$, which we use as the control parameter.

\section{RESULTS AND DISCUSSION}

In Fig. 1 we present an overview of the types of behavior as a function of the vorticity $\mu_{2}$ in the form of $24 x-t$ of $|u|$ plots for $\mathrm{T}=2000$. We note that due to the large scale one does not see in Fig. 1 any difference whether one plots $|u|$ or
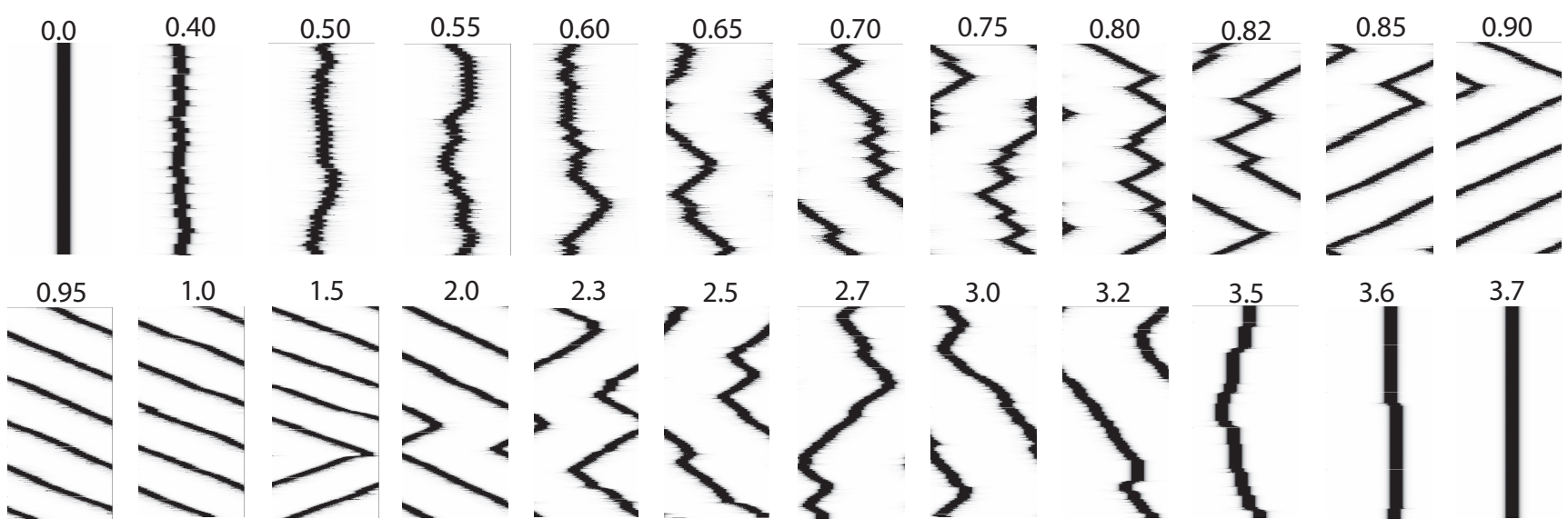

FIG. 1. $x-t$ plots for $|u|$ of the types of behavior observed as a function of $\mu_{2}$ for the interval $0 \leqslant \mu_{2} \leqslant 3.7$. For each $x-t$ plot $T=2000$ and box size $L=50$. The sequence of these plots shows the transition from a localized oscillatory state $\left(\mu_{2}=0\right)$ via nonmoving exploding DS (at $\mu_{2}=0.4$ ) to propagating DS and then back to oscillatory localized solutions via exploding DS. 
the modulus $R=\sqrt{u^{2}+v^{2}}$. As $\mu_{2}$ is increased from 0 , we observe at $\mu_{2}=0.35$ a transition from nonmoving oscillatory localized solutions to exploding dissipative solitons showing a meandering motion with net velocity zero when averaged over long times. This regime is followed by exploding dissipative solitons with frequent direction changes (compare, for example, $\mu_{2}=0.75$ ) and eventually there are propagating exploding dissipative solitons propagating in only one direction for times at least as long as $T>2 \times 10^{4}$. As $\mu_{2}$ is increased further, the inverse sequence of transitions is observed and for $\mu_{2}=3.7$ nonmoving oscillatory localized solutions are recovered.

While exploding dissipative solitons have been found for the cubic-quintic CGL equation in one and two spatial dimensions, these were not moving on an average, but showed the meandering motion with average velocity zero also encountered for the present reaction-diffusion system. As the cubic-quintic complex CGL equation has much higher symmetry than the RD system studied here, we attribute the existence of propagating exploding dissipative solitons for long times to the reduced symmetry. For example, when incorporating a vorticity of the type $\sim \mu_{2}$ into the cubicquintic CGL equation, this additional linear coupling could be transformed away for the noise-free cubic-quintic CGL equation by going into a rotating frame. For the RD system studied here the same transformation would lead to a number of additional nonlinearities.

To characterize several of the states observed in more detail, we show in Figs. 2(a) and 2(b) snapshots for $u$ and $v$ for $\mu_{2}=0.45$. We have incorporated as a solid line as a guide for the eye $R=\sqrt{u^{2}+v^{2}}$. While this quantity is not directly measurable for a chemical reaction, it facilitates the comparison with the results obtained from the cubicquintic CGL equation for exploding dissipative solitons. In Figs. 2(c)-2(e), we show three short $x-t$ plots $(T=400)$ for $|u|$ revealing the details of the characteristic behavior of the types of exploding dissipative solitons observed for our RD system. Figure 2(c) shows $|u|$ for a meandering exploding dissipative soliton at $\mu_{2}=0.45$ and is very reminescent of what one finds for $R$ for exploding dissipative solitons in the cubic-quintic CGL equation. This changes drastically when increasing $\mu_{2}$ to $\mu_{2}=0.8$ [Fig. 2 (d)]. Now the exploding dissipative soliton is propagating over distances that become comparable to the box size before a change in direction while for $\mu_{2}=0.98$ the propagation distance is already larger than the box size [Fig. 2 (e)]. We emphasize that Figs. 2(c)-2(e) are the short time analogues of the $24 x-t$ plots shown in Fig. 1 for $T=2000$ each.

To study the behavior of the propagation distance as a function of $\mu_{2}$ we have made systematically long runs for the interval $0.90 \leqslant \mu_{2} \leqslant 1.05$. In Fig. 3 we show the results for $\mu_{2}=0.95$ on the left [Fig. 3 (a)] and for $\mu_{2}=0.98$ on the right [Fig. 3 (b)]. In both cases we show the asymptotic time regime for $T=2 \times 10^{4}$. The picture that emerges from our long runs is that there is an interval for $\mu_{2}$ for which one has propagation distances that are very large.

In Fig. 4 we have plotted the number of direction changes, $N$, as well as the various regimes described above as a function of $\mu_{2}$. At $\mu_{2}=0.35$ there is an intermittent transition from oscillatory to meandering, which is intrinsi-
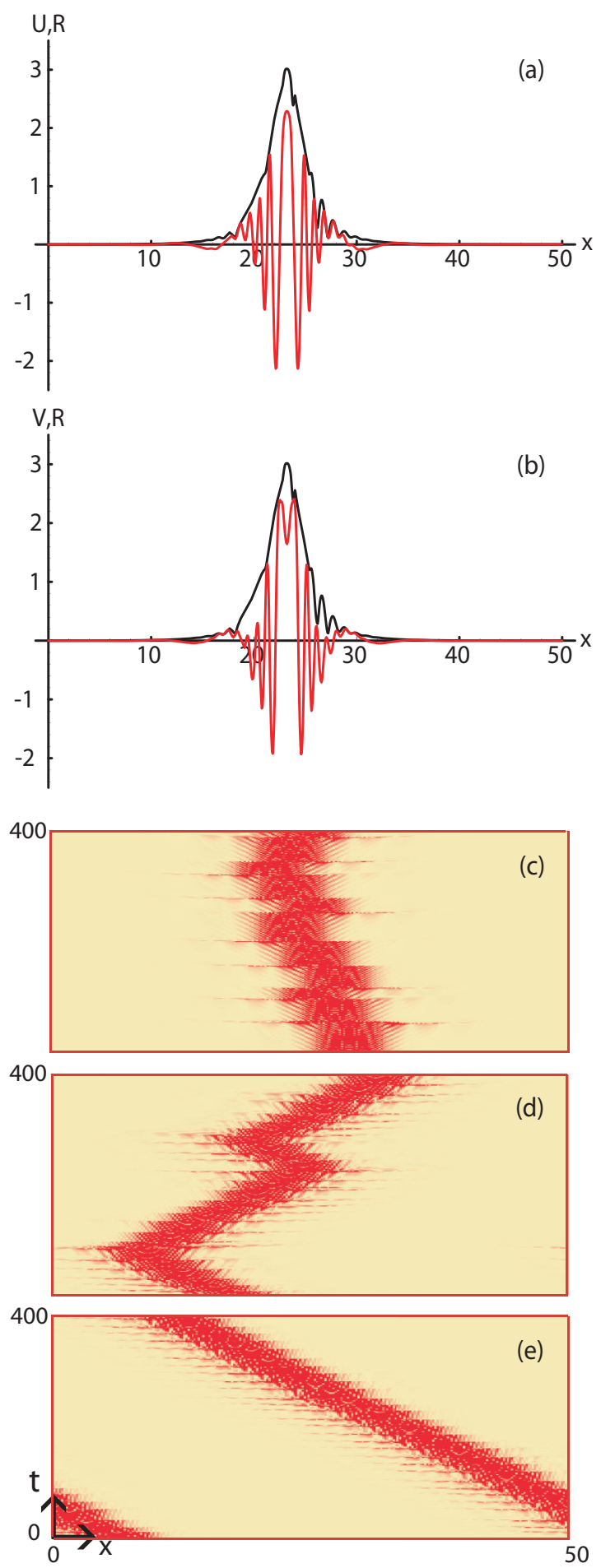

FIG. 2. (Color online) (a) and (b) show in red snapshots of $u(x, t)$ and $v(x, t)$ for $\mu_{2}=0.45$ for the regime of meandering exploding dissipative solitons. The solid black lines correspond to the modulus $R=\sqrt{u^{2}+v^{2}}$; (c), (d), and (e) show short $x-t$ plots $(\mathrm{T}=400)$ of $|u|$ for $\mu_{2}=0.45$ (meandering explosions), $\mu_{2}=0.8$ (frequent changes in propagation direction), and $\mu_{2}=0.98$ (propagation in one direction for long times).

cally connected to explosions. We note that oscillations in the wings come together with explosions. For $\mu_{2}=3.7$ we 


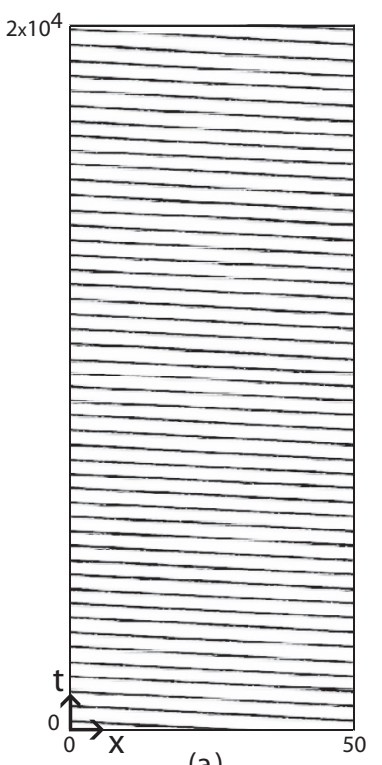

(a)

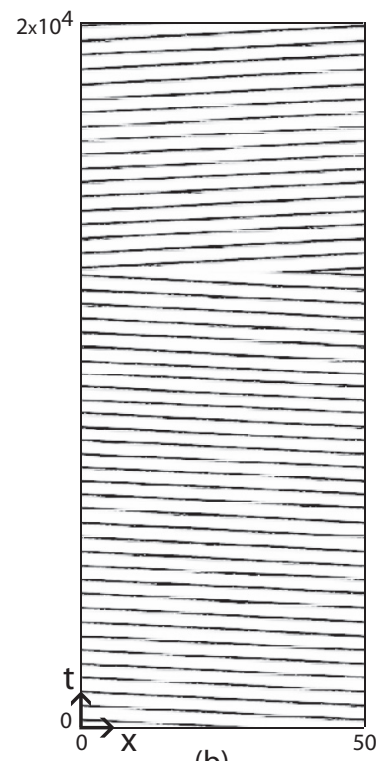

(b)
FIG. 3. $x-t$ plots for $T=2 \times 10^{4}$ showing the asymptotic behavior for (a) $\mu_{2}=0.95-$ no change in propagation directionand (b) $\mu_{2}=0.98$ - one change in propagation direction.

find the corresponding reverse transition. We emphasize that for the present RD system the sequence of explosions is not simply alternating LRLRLR, etc., as for the cubic-quintic CGL equation. We attribute this difference to the lower symmetry of our RD system. As a consequence of this deviation from a strict alternation, one obtains the meandering exploding dissipative solitons of the type shown in Fig. 2(c). This gives for short times the impression of a mean drift. However, when waiting long enough-compare, for example, the $x-t$ plots for $\mu_{2}=0.55,0.60$, and $\mu_{2}=3.5$ in Fig. $1-$ there is no net drift in the meandering regime. For the exploding dissipative solitons in the cubic-quintic CGL equation the time over which one has to average to obtain no net drift is considerably shorter $[36,40]$. For the parameter range $0.90 \leqslant \mu_{2} \leqslant 2.2$, there are direction changes only as rare events (IDC), which require sufficiently large fluctuations as a trigger.

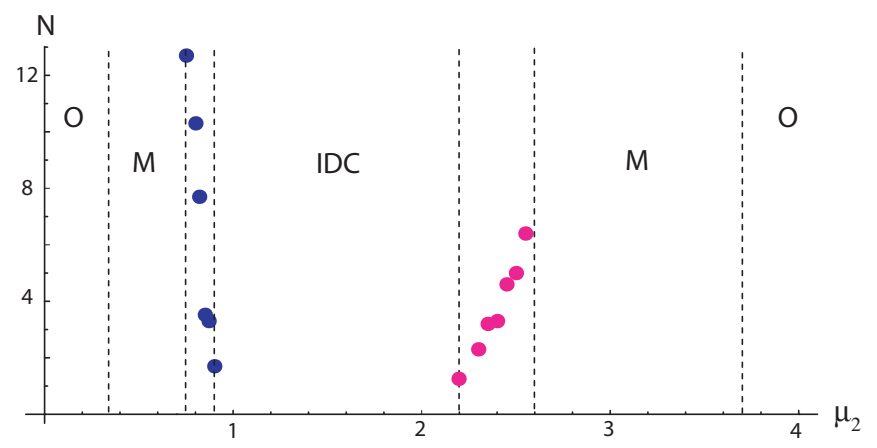

FIG. 4. (Color online) The number of direction changes, $N$, is plotted as a function of $\mu_{2}$ for $T=2 \times 10^{3}$, where the values of $N$ represent averages over at least six runs. $\mathrm{O}$ denotes oscillatory behavior, $\mathrm{M}$ stands for meandering, and IDC stands for infrequent direction changes.

\section{DIFFERENCES AND SIMILARITIES OF DISSIPATIVE SOLITONS IN THE RD SYSTEM VERSUS IN THE CUBIC-QUINTIC CGL EQUATION}

In this section we analyze differences and similarities of various types of dissipative solitons for the RD system investigated here in comparison with the properties of such solutions for the only other equation for which exploding dissipative solitons have been studied so far: the cubic-quintic CGL equation. Close to the onset of an instability it takes the form

$$
\begin{aligned}
\partial_{t} A= & \left(\mu+i \mu_{2}\right) A+\left(\beta_{r}+i \beta_{i}\right)|A|^{2} A+\left(\gamma_{r}+i \gamma_{i}\right)|A|^{4} A \\
& +\left(D_{r}+i D_{i}\right) A_{x x},
\end{aligned}
$$

where we have written down explicitly also the imaginary part of the linear growth term, $\mu_{2}$, for comparison purposes with the RD system (compare the analysis given further below). We note that the mapping from the instability version of the cubic-quintic CGL equation to the notation used in optics [35] has been discussed in detail in Ref. [40]. Splitting the complex scalar envelope function $A$ into real and imaginary parts, $A=u+i v$, we have

$$
\begin{aligned}
\partial_{t} u= & \mu u-\mu_{2} v+\beta_{r} u\left(u^{2}+v^{2}\right)+\gamma_{r} u\left(u^{2}+v^{2}\right)^{2} \\
& -\beta_{i} v\left(u^{2}+v^{2}\right)-\gamma_{i} v\left(u^{2}+v^{2}\right)^{2} \\
& +D_{r} u_{x x}-D_{i} v_{x x}, \\
\partial_{t} v= & \mu v+\mu_{2} u+\beta_{r} v\left(u^{2}+v^{2}\right)+\gamma_{r} v\left(u^{2}+v^{2}\right)^{2} \\
& +\beta_{i} u\left(u^{2}+v^{2}\right)+\gamma_{i} u\left(u^{2}+v^{2}\right)^{2} \\
& +D_{r} v_{x x}+D_{i} u_{x x} .
\end{aligned}
$$

This is a formulation of the cubic-quintic CGL equation, which can be directly compared with the reaction diffusion model studied in the present paper [Eqs. (1) and (2)]:

$$
\begin{gathered}
\partial_{t} u=\mu_{1} u-\mu_{2} v+\beta_{1} u^{3}+\gamma_{1} u^{5}-\beta_{2} v^{3}-\gamma_{2} v^{5} \\
+D_{r} u_{x x}-D_{i} v_{x x}, \\
\partial_{t} v=\mu_{3} v+\mu_{2} u+\beta_{1} v^{3}+\gamma_{1} v^{5}+\gamma_{2} u^{5} \\
\quad+D_{r} v_{x x}+D_{i} u_{x x} .
\end{gathered}
$$

From the direct comparison of the two systems a number of differences emerge immediately:

(1) The cubic-quintic CGL equation has global gauge invariance, that is the equation is invariant under $A \rightarrow$ $A \exp (i \varphi)$ with a constant phase $\varphi$. This invariance is absent in the RD model studied.

(2) The nonlinearities in the cubic-quintic CGL equation are of the form $\left(u^{2}+v^{2}\right)^{n} u$ and $\left(u^{2}+v^{2}\right)^{n} v$ with $n=1,2$. The nonlinearities of the RD model are qualitatively different in nature and are of much lower symmetry.

(3) The coefficient $\beta_{2}$ only occurs in one equation in contrast to the cubic-quintic CGL equation where all coefficients appear in both equations.

(4) The coefficient $\mu_{2}$ in the cubic-quintic CGL equation can be transformed out of the equation via $A=\tilde{A} \exp \left(i \mu_{2} t\right)$. A transformation that is usually done. This transformation leaves the structure of the cubic-quintic CGL equation invariant. Applying such a transformation to the RD system one 
obtains equations with nonlinearities that have in addition an explicit time dependence. Thus leading to no simplification of the problem and no invariance property. As a direct consequence, none of the figures presented in this paper as a function of $\mu_{2}$ can be obtained for the cubic-quintic CGL equation.

(5) The reactive part of both systems (discarding diffusion effects) has different dynamical behavior. Besides the fixed point at the origin, which is an attractor (A), the RD system has saddle nodes $(\mathrm{SN})$ and repellers $(\mathrm{R})$ as fixed points, as is shown in Figs. 5(a) and 5(b). The number of fixed points changes as a function of $\mu_{2}$. In the cubic-quintic CGL equation, the only fixed point is the origin [see Fig. 5(c)]. In addition, both systems have a limit cycle (not shown in the figure) as an attractor.

These differences have also a number of other consequences. For example, the fixed-shape solution for the cubic-quintic CGL equation is replaced by an oscillating dissipative soliton with oscillations around a fixed shape. This has already been discussed for a somewhat simpler RD model in Refs. [49-52].

The reduction of symmetry discussed above has several other consequences, including the possibility of having exploding propagating DSs for long times only in the RD system as well as the fact that the direction changes of the exploding DSs are strictly LRLRLRLR... for the cubic-quintic CGL equation while for the RD system this strictly alternating behavior is absent (compare also the remark at the end of the last section).

To conclude this section, we also note some similarities between the two different systems. We obtain exploding DSs for anomalous linear dispersion, $D_{i}$, in both cases. For exploding DSs we need furthermore two nonvariational nonlinearities in both types of model. The intuitive picture we have in this connection is that we need the possibility to generate an instability in the wings of the exploding DS. In addition, one must guarantee that the localized solution does not expand and fill the system completely.

\section{CONCLUSIONS AND PERSPECTIVE}

In conclusion, we demonstrated that two-component RD systems have solutions in the form of exploding dissipative solitons. They exist for a certain region of parameters. Numerical simulations allowed us to find them when we varied one of the parameters while the others have been fixed. Several boundaries of different dynamics for the different types of dissipative solitons have been identified.

Here we have shown that three parameters play an important role to have exploding dissipative solitons over a range of parameters: $\beta_{2}$, the prefactor of the nonvariational $v^{3}$ term in the equation for $u, \gamma_{2}$, the prefactor of the nonvariational terms in the equations for $u$ and $v$ and $D_{i}$, the linear dispersion. This has to be contrasted to the requirements to obtain dissipative solitons stably in the reaction-diffusion systems. Previous investigations [49-52] on a simple RD system with two variables have shown that one nonvariational parameter associated with a nonlinearity is sufficient to obtain particle as well as several types of hole solutions stably. Thus, exploding
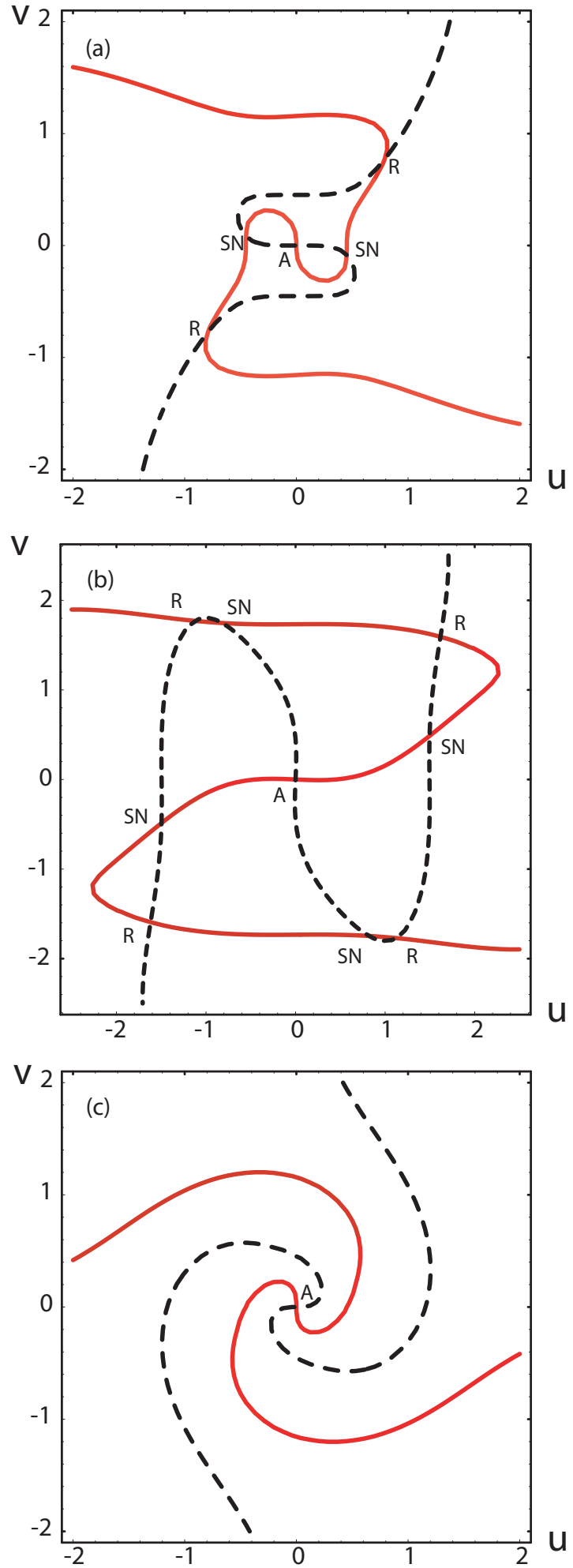

FIG. 5. (Color online) (a) Nullclines of the RD system for $\mu_{2}=$ 0.0 . The solid line denotes $\partial_{t} u=0$ and the dashed line $\partial_{t} v=0$ for Eqs. (1) and (2). Besides the attractor at the origin (A), there are two saddle nodes ( $\mathrm{SN}$ ) and two repellers (R) as fixed points. (b) Nullclines of the RD system for $\mu_{2}=4.5$. Besides the attractor at the origin (A) there are four saddle nodes (SN) and four repellers (R) as fixed points. (c) Nullclines of the cubic-quintic CGL equation. The only fixed point is the origin. 
dissipative solitons clearly emerge as a more complex object than dissipative solitons of the usual type. This is also brought out by our studies on the cubic-quintic CGL equation, which also revealed that one needs essentially nonzero values in a certain range for the two nonvariational parameters $\gamma_{i}$ and $\beta_{i}$ and the linear dispersion $D_{i}$ to obtain exploding dissipative solitons, while for simple one-particle solutions, one nonvanishing nonvariational parameter has turned out to be sufficient for stabilization.

Clearly, our present study opens the door to several areas of investigation. First of all, it will be important to see which features found for exploding dissipative solitons in RD systems in one spatial dimension can be generalized to two spatial dimensions and which additional phenomena might occur. This question has been recently addressed for the cubic-quintic CGL equation [45],

We also expect the investigation of the influence of noise on exploding dissipative solitons in RD systems to be a promising line of future research, all the more since noise is ubiquitous in biological systems that are frequently modeled by RD systems. Our previous studies on the influence of noise on the transition to exploding dissipative solitons in the cubic-quintic CGL equation have already revealed rather complex response behavior even to fairly small noisy perturbations [42].

Naturally, our recent study on the transition from inanimate matter to localized solutions showing elementary features of life obtained for the combination of a reaction diffusion system for matter flow and of a cubic-quintic CGL equation for energy flow [7] deserves a generalization along the lines given here to investigate to what extent exploding dissipative solitons are relevant in a biological context.

An outstanding challenge is certainly to find an experimental system of RD-type (chemical or biological) that shows exploding dissipative solitons. While this program has been carried out successfully for the cubic-quintic CGL equation in nonlinear optics [37], we are not aware as yet of any experimental work done for RD systems. Studies of impulses in biological membranes demonstrate complicated explosionlike behavior of current pulses [53,54] when ionic content is changed. Hardware neuron model simulations explain this behavior in terms of "bursts" [55,56]. However, bursts are a relatively simple chaotic increase of the pulse amplitudes observed after bifurcations. Propagation effects may turn these "bursts" into explosions. Study of these effects may require more complicated equipment with spectral recording as in Ref. [37], instead of simply observing the time dependence. Our present study could stimulate work in this direction.

\section{ACKNOWLEDGMENTS}

O. D. acknowledges the support of FONDECYT (Project No. 1110360) and Universidad de los Andes through FAI initiatives. N. A. thanks the Australian Research Council (Discovery Project No. DP110102068). N. A. is supported by an Alexander von Humboldt Research Prize. H. R. B. thanks the Deutsche Forschungsgemeinschaft for partial support of this work.
[1] M. C. Cross and P. C. Hohenberg, Rev. Mod. Phys. 65, 851 (1993).

[2] M. Tlidi, R. Lefever, and A. Vladimirov, Lecture Notes in Physics, Vol. 751 (Springer, Heidelberg, 2008), p. 381.

[3] H. Li, D. G. Ouzounov, and F. W. Wise, Opt. Lett. 35, 2403 (2010).

[4] Ph. Grelu and N. Akhmediev, Nature Photonics 6, 84 (2012).

[5] N. Akhmediev and A. Ankiewicz, Editors, Dissipative Solitons: From Optics to Biology and Medicine (Springer, Heidelberg, 2008).

[6] S. M. Mohseni et al., Science 339, 1295 (2013).

[7] N. Akhmediev, J. M. Soto-Crespo, and H. R. Brand, Phys. Lett. A 377, 968 (2013)

[8] T. Ohta, Y. Hayase, and R. Kobayashi, Phys. Rev. E 54, 6074 (1996).

[9] J. Kosek and M. Marek, Phys. Rev. Lett. 74, 2134 (1995).

[10] K. J. Lee, W. D. McCormick, Q. Ouyang, and H. L. Swinney, Nature (London) 369, 215 (1994).

[11] W. N. Reynolds, J. E. Pearson, and S. Ponce-Dawson, Phys. Rev. Lett. 72, 2797 (1994).

[12] Y. Hayase and T. Ohta, Phys. Rev. Lett. 81, 1726 (1998).

[13] H. R. Brand, P. S. Lomdahl, and A. C. Newell, Physica D 23, 345 (1986); Phys. Lett A 118, 67 (1986).

[14] O. Thual and S. Fauve, J. Phys. France 49, 1829 (1988).

[15] H. R. Brand and R. J. Deissler, Phys. Rev. Lett. 63, 2801 (1989).
[16] R. J. Deissler and H. R. Brand, Phys. Rev. A 44, R3411 (1991).

[17] R. J. Deissler and H. R. Brand, Phys. Rev. Lett. 72, 478 (1994).

[18] H. R. Brand and R. J. Deissler, Physica A 204, 87 (1994).

[19] R. J. Deissler and H. R. Brand, Phys. Rev. E 51, R852 (1995).

[20] R. J. Deissler and H. R. Brand, Phys. Rev. Lett. 74, 4847 (1995).

[21] H. R. Brand and R. J. Deissler, Physica A 216, 288 (1995).

[22] V. V. Afanasjev, N. Akhmediev, and J. M. Soto-Crespo, Phys. Rev. E 53, 1931 (1996).

[23] O. Descalzi and H. R. Brand, Phys. Rev. E 72, 055202 (2005).

[24] O. Descalzi, J. Cisternas, D. Escaff, and H. R. Brand, Phys. Rev. Lett. 102, 188302 (2009).

[25] P. Kolodner, Phys. Rev. A 44, 6448 (1991).

[26] P. Kolodner, Phys. Rev. A 44, 6466 (1991).

[27] H. H. Rotermund, S. Jakubith, A. von Oertzen, and G. Ertl, Phys. Rev. Lett. 66, 3083 (1991).

[28] P. B. Umbanhowar, F. Melo, and H. L. Swinney, Nature (London) 382, 793 (1996).

[29] O. Lioubashevski, Y. Hamiel, A. Agnon, Z. Reches, and J. Fineberg, Phys. Rev. Lett. 83, 3190 (1999).

[30] P. Tsai, S. W. Morris, and Z. A. Daya, Eur. Phys. Lett. 84, 14003 (2008).

[31] V. B. Taranenko, K. Staliunas, and C. O. Weiss, Phys. Rev. A 56, 1582 (1997).

[32] G. Slekys, K. Staliunas, and C. O. Weiss, Opt. Commun. 149, 113 (1998). 
[33] E. A. Ultanir, G. I. Stegeman, D. Michaelis, C. H. Lange, and F. Lederer, Phys. Rev. Lett. 90, 253903 (2003).

[34] K. Staliunas and V. J. Sánchez-Morillo, Transverse Patterns in Nonlinear Optical Resonators, Springer Tracts in Modern Physics, Vol. 183 (Springer, Berlin, 2003).

[35] J. M. Soto-Crespo, N. Akhmediev, and A. Ankiewicz, Phys. Rev. Lett. 85, 2937 (2000).

[36] N. Akhmediev, J. M. Soto-Crespo, and G. Town, Phys. Rev. E 63, 056602 (2001).

[37] S. T. Cundiff, J. M. Soto-Crespo, and N. Akhmediev, Phys. Rev. Lett. 88, 073903 (2002).

[38] N. Akhmediev and J. M. Soto-Crespo, Phys. Lett. A 317, 287 (2003).

[39] N. Akhmediev and J. M. Soto-Crespo, Phys. Rev. E 70, 036613 (2004).

[40] O. Descalzi and H. R. Brand, Phys. Rev. E 82, 026203 (2010).

[41] O. Descalzi, C. Cartes, J. Cisternas, and H. R. Brand, Phys. Rev. E 83, 056214 (2011).

[42] C. Cartes, O. Descalzi, and H. R. Brand, Phys. Rev. E 85, 015205 (2012).

[43] A. Ankiewicz, N. Devine, N. Akhmediev, and J. M. Soto-Crespo, Phys. Rev. A 77, 033840 (2008).
[44] J. M. Soto-Crespo, N. Akhmediev, N. Devine, and C. MejiaCortes, Opt. Express 16, 15388 (2008).

[45] C. Cartes, J. Cisternas, O. Descalzi, and H. R. Brand, Phys. Rev. Lett. 109, 178303 (2012).

[46] P. C. Hohenberg and J. B. Swift, Phys. Rev. A 46, 4773 (1992).

[47] K. Staliunas, Phys. Rev. E 64, 066129 (2001).

[48] H. Sakaguchi and H. R. Brand, Physica D-Nonlinear Phenomena 97, 274 (1996).

[49] Y. Hayase, O. Descalzi, and H. R. Brand, Phys. Rev. E 69, 065201 (2004).

[50] Y. Hayase, O. Descalzi, and H. R. Brand, Physica A 356, 19 (2005).

[51] O. Descalzi, Y. Hayase, and H. R. Brand, Int. J. Bifurcation Chaos Appl. Sci. Eng. 14, 4097 (2004).

[52] O. Descalzi, Y. Hayase, and H. R. Brand, Phys. Rev. E 69, 026121 (2004).

[53] M. Deschênes, J. P. Roy, and M. Steriade, Brain Res. 239, 289 (1982).

[54] H. Jahnsen and R. Llinás, J. Physiol. - London 349, 227 (1984).

[55] Y. Maeda, H. Makino, BioSystems 58, 93 (2000).

[56] M. Ciszak, S. Euzzor, A. Geltrude, K. Al-Naimee, F. T. Arecchi, and R. Meucci, Cybernetics and Physics 1, 22 (2012). 